\title{
Early stage temperature conditions influence on micromechanical features of thermoset polymer-based composite
}

\author{
Jan Vorel ${ }^{1}$, Vladimír Hrbek ${ }^{1}$, Michal Šejnoha ${ }^{1}$ \\ $1^{1} \mathrm{CTU}$ in Prague, Faculty of Civil Engineering, Department of Mechanics, Czech Republic \\ Email: jan.vorel@fsv.cvut.cz
}

The application of thermoset polymer-based composites is widespread in many engineering fields, such as automotive, aerospace or bioengineering industry. These composites found their civil engineering utilisation, e.g., as post-installed anchoring systems or in concrete and masonry reconstructions. Regardless of the use, environmental conditions imposed on a typical thermoset polymer matrix during casting and early curing stage predetermine properties of the composite in a hardened state. Moreover, unstable environmental conditions that usually occur in civil engineering practice may lead to structural service life reduction or, in the worst case, abrupt failure due to alternated mechanical features.

In general, the modelling and optimisation of polymer-based composites are decisively dependent on understanding two essential aspects; heat generation during the polymer's initial chemical reaction and the material formation reflected in a strength gain. The contribution presents an experimental evaluation of thermoset polymer-based composite, which was cast and cured at different temperatures, to capture initial environmental fluctuance. The reaction heat generation is monitored by using isothermal calorimetry. Moreover, both viscoelasticity and strength gains are measured on the material microscale using nano-indentation. Supported by described experimental work, bottom-up uncoupled multi-scale homogenisation is used to estimate temperature impact on material properties. Furthermore, the data can be applied in curing kinetics and heat generation models or stress evolution and structural integrity development simulations.

\section{Acknowledgement}

The financial support provided by the Grant Agency of Czech Republic grant No. 19-15666S is gratefully acknowledged. 